\section{¿Qué muestran las Encuestas de Calidad de Vida sobre el sistema de salud en Colombia?}

\author{
What do living standard surveys show about the \\ health system in Colombia?
}

\footnotetext{
${ }_{1}^{1}$ Facultad de Economía, Universidad del Rosario, Bogotá, Colombia.

2 Asociación Colombiana de la Salud, Bogotá, Colombia.

Correspondencia A. Zambrano

Facultad de Economía. Universidad del Rosario. Calle 14 \# 4-69, Bogotá, Colombia.

jzambran@urosario.edu.co
}

\section{Abstract}

This paper analyzes the main advances in health coverage and health services demand in Colombia using the 1997 and 2003 living standards surveys. The study showed an increase in health coverage associated with expansion of the subsidized care system, although a large proportion of the population is still not entitled to health care. The type of entitlement also directly affects the demand for services. There was an increase in preventive medical consultations in 2003 and a decrease in the use medical care for acute illness, due mainly to lack of money. Access to medicines increased from 1997 to 2003, as reflected by a decrease in out-of-pocket expenditure on medicines.

Health Services Coverage; Health Services Needs and Demand; Quality of Life

\author{
Andrés Zambrano ${ }^{1}$ \\ Manuel Ramírez 1 \\ Francisco José Yepes 2 \\ José Alberto Guerra 1 \\ David Rivera 1
}

\section{Introducción}

El primero de enero de 1995 entró en vigencia en Colombia una profunda reforma del sistema de seguridad social en salud (que había sido aprobada por ley en 1993), la cual pretendía el aseguramiento universal en salud con eficiencia, calidad y acceso a los servicios de salud. Establecía dos regímenes de aseguramiento: uno contributivo para quienes tienen un empleo formal o la forma de pagar y uno subsidiado para los pobres.

La última información disponible sobre cobertura del aseguramiento antes de dicha reforma nos informa que durante el período 19861989 existe una cobertura de la seguridad social en salud del $23,8 \%$, que sumada a la de otros tipos de seguros alcanzaba al 30,4\% de la población 1 . La población pobre no asegurada debía acceder a la red pública de servicios de salud.

Según los resultados de las Encuestas de Calidad de Vida de 1993 y 1997 2, la reforma del sistema de salud a través de la Ley 100 produjo avances significativos. El porcentaje de afiliados en 1997 pasó al 57\%; el régimen subsidiado en 1997 cubría a 7.681 .550 personas de bajos recursos, y el contributivo atendía a 15.072.983 colombianos. En 2003 la afiliación era de 61,8\%, algo más de 17 millones en el contributivo y 10 millones en el subsidiado.

A pesar del aumento de la afiliación, se continuaban presentando diferencias significativas en características sociodemográficas y geográficas. 
Por ejemplo, las áreas rurales y centros poblados mostraron índices de cobertura de servicios por debajo de las cabeceras municipales. De hecho, el régimen subsidiado se presenta en las zonas rurales como el mecanismo más importante de aseguramiento en el 2003, con una afiliación del $35,5 \%$ de las personas del área rural, mientras que el régimen contributivo cubre al $12 \%$. Algunas de estas diferencias geográficas y otras sociodemográficas persisten en la actualidad.

El propósito principal de un sistema de salud es mejorar la salud de las personas. La estrategia adoptada por Colombia ha sido la de basar el sistema de salud en el aseguramiento de sus ciudadanos, y a partir de allí prestar servicios médicos que mejoren la salud de éstos. Por lo tanto, examinar las variables que están relacionadas con el aseguramiento y cómo, a su vez, el aseguramiento se relaciona con la demanda de servicios médicos, es un componente importante para la evaluación del sistema de salud. El objetivo de este artículo es el de evaluar el desempeño del sistema de salud después de la reforma hecha en 1993, a través de los avances en cobertura, medida a través del aseguramiento, y la demanda de servicios médicos en Colombia.

Para el cumplimiento de este objetivo utilizamos las Encuestas de Calidad de Vida de 1997 (ECV97) y 2003 (ECV03), realizadas por el Departamento Administrativo Nacional de Estadística (DANE). El diseño de estas encuestas también ayuda a comparar la evolución de las principales variables en dos momentos del tiempo interesantes desde el punto de vista económico, en el primero de ellos Colombia se encontraba en el preámbulo de una recesión económica y en el segundo la crisis estaba siendo superada. En este punto es importante resaltar que el propósito será el de analizar los resultados de la reforma antes de la introducción de las nuevas modificaciones hechas a partir de 2005, aunque también se hacen algunas consideraciones relativas a estos nuevos cambios.

Entre los resultados más destacados se encuentra un aumento del aseguramiento, que aún no ha sido suficiente para cubrir a los más vulnerables, y una relación positiva entre el aseguramiento y la demanda de servicios médicos, aunque esta última se vio afectada negativamente por la disminución en la capacidad adquisitiva de los individuos. El desarrollo del artículo será de la siguiente manera: en la segunda sección se expondrán aspectos metodológicos, en una tercera sección se mostrarán los principales resultados derivados de la estadística descriptiva y, por último, se enumerarán algunas conclusiones donde se discuten los efectos de algunas políticas implementadas recientemente.

\section{Consideraciones metodológicas}

Las ECV97 y ECV03 son ideales para la comparación intertemporal de diversas variables socioeconómicas por la abundancia de este tipo de preguntas, porque la mayoría de ellas son perfectamente comparables y porque el diseño muestral es muy similar. A continuación describiremos las variables utilizadas en el análisis y las aproximaciones utilizadas para homogeneizarlas cuando éstas difieran entre las dos encuestas, de manera que puedan realizarse comparaciones que sean válidas y confiables. También se enunciarán las diferencias que existen entre las dos encuestas en términos del marco muestral.

Para evaluar el sistema de salud se analizan la relación entre el aseguramiento y variables sociodemográficas y geográficas, y la demanda de servicios de salud de acuerdo con el tipo de afiliación; en ambos casos también se hacen comparaciones intertemporales. Cuando las variables son categóricas se estudian a través de probabilidades condicionales y tablas de contingencia, y si son continuas a través de sus respectivas medias; en todos los casos se evalúan estadísticamente sus diferencias.

El aseguramiento es analizado a partir del tipo de afiliación y la pertenencia a planes complementarios de salud. Como planes complementarios de salud se consideran las pólizas de hospitalización y cirugía, la medicina prepagada y los seguros estudiantiles, entre otros. El tipo de afiliación es discriminado en afiliado al régimen contributivo, afiliado al régimen subsidiado y no afiliado. Dado que las preguntas relativas al tipo de afiliación son distintas entre la ECV97 y la ECV03, a continuación se describe la construcción de esta variable.

Para 2003, se considera que pertenecen al régimen contributivo las personas afiliadas al Instituto Nacional de Seguros Sociales (ISS), Caja Nacional de Previsión (CAJANAL) y otras Entidad Promotora de Salud (EPS): fuerzas militares, Policía Nacional, Ecopetrol y Magisterio. Además, se tomaron las personas que estaban afiliadas a otras entidades y no hubieran sido inscritas a través del Sistema de Selección de Beneficiarios de Programas Sociales (SISBEN). Por su parte, se consideran del régimen subsidiado las personas que pertenezcan a las administradoras del régimen subsidiado (ARS), empresas solidarias y las inscritas en otras entidades que hubieran sido afiliadas por el SISBEN.

Para 1997, se consideran del régimen contributivo las personas que estén cubiertas por una entidad de seguridad social y sean pensionados; trabajadores cuya afiliación la paguen ellos o sus empresas; familiares de un afiliado a una EPS, 
CAJANAL, fuerzas militares, Policía Nacional, Magisterio o Ecopetrol que no tenga carné del SISBEN. Se consideran del régimen subsidiado las personas que estén cubiertas por una entidad de seguridad social o Caja de Compensación y no paguen por dicha afiliación o sean familiares de un afiliado a una empresa solidaria $u$ otra.

Las personas que no están cubiertas por alguna entidad de seguridad social en salud y por ende no pertenecen a ninguno de los anteriores regímenes, se denominan no asegurados. El DANE 2 caracteriza esta población en tres subgrupos. El primer grupo está compuesto por personas pobres que no son identificadas por medio del SISBEN; el segundo incluye a quienes a pesar de tener ingresos suficientes para cotizar en el régimen contributivo no lo hacen por falta de incentivos; el tercero involucra a las personas que no se encuentran propiamente en situación de vulnerabilidad, pero que no disponen de ingresos suficientes para pagar una cotización en el régimen contributivo.

Entre las variables geográficas se manejan la región y la zona de residencia del hogar (urbano o rural). Entre las variables socioeconómicas tomamos los quintiles de ingreso y la informalidad en el trabajo. Un individuo se cataloga como informal si su régimen de salud no es contributivo, habita en cabeceras municipales y cumple una de las siguientes condiciones: (a) es trabajador independiente con menos de once años de educación, (b) es patrón o empleado particular de una empresa compuesta por menos de diez personas, (c) es un trabajador familiar sin remuneración, empleado doméstico o jornalero (peón). Esta definición es la misma utilizada por el DANE para la Encuesta Continua de Hogares.

Para aproximarnos a la demanda de servicios médicos examinamos la proporción de personas que estando sanas consultaron al médico $\mathrm{u}$ odontólogo por prevención por lo menos una vez al año, la proporción de personas que sintiéndose enfermas en el último mes consultaron al médico y la proporción de personas que usaron medicamentos en el último mes y su respectivo gasto en éstos. Con respecto a la consulta cuando se percibe enfermedad, se examinó también las razones por las cuales el individuo no acudió al médico con el fin de evaluar la importancia del dinero en esta decisión.

Con respecto a la demanda de servicios médicos también existen algunas consideraciones metodológicas que vale la pena mencionar. La demanda por hospitalizaciones no fue analizada porque las preguntas cambiaron sustancialmente entre las encuestas. Otro tipo de análisis, como exámenes de diagnóstico o gasto en rubros como vacunas terapias o transporte, tampoco se puede realizar porque en la encuesta del 2003 no se encuentra información sobre estos temas.

Por su parte, la proporción de personas que se sintieron enfermas en el último mes no es comparable entre los dos años por cambios en el flujo de preguntas, porque las encuestas se realizaron en periodos distintos del año y por la subjetividad en la percepción; así que no es sensato interpretar las diferencias de estas preguntas en ambos años. Muestra de esto es que para $1997 \mathrm{el}$ porcentaje de personas que revelaron tener un problema de salud fue de $16,2 \%$, mientras para el 2003 esta proporción fue de 11,5\%. Sin embargo, sí se pueden comparar las preguntas relativas a qué acciones tomaron para curar el problema de salud y las razones por las que no utilizaron los servicios médicos, pues son las mismas en ambos años.

Con respecto al gasto en medicamentos, la ECV97 indaga sobre el gasto mensual de cada persona en medicamentos o remedios, mientras que la ECV03 se pregunta por el gasto mensual del hogar en fórmulas médicas o compra de medicinas consumidas regularmente. Con el fin de hacer las preguntas comparables, para 1997 se calculó el gasto del hogar en medicamentos, agregando el de todos los miembros del hogar, luego se dividió por el número de personas del hogar y se asignó este gasto a cada uno. Para 2003 se dividió el gasto del hogar por el número de personas y de nuevo se asignó este gasto a cada uno. Es necesario tener en cuenta que esta aproximación genera sesgos. Puede pasar que una única persona en el hogar consumió las medicinas y con este método se le imputa a cada miembro del hogar una parte del gasto de ésta, esto genera que el promedio del gasto tienda a ser menor que el real. Sin embargo, si se trabajara el consumo de medicinas en el hogar se tendría que dentro de un mismo hogar pueden vivir personas afiliadas a distintos regímenes y habría que elegir algún criterio para considerar el hogar en algún régimen.

Las diferencias en términos muestrales entre las dos encuestas son menores. La encuesta de 2003 considera las mismas regiones que la de 1997, pero la región Pacífica se dividió en dos: Valle y el resto la región Pacífica, aumentando así el número de observaciones. También en 2003 hubo un aumento importante de la muestra en Bogotá para lograr la significancia por localidades. Ninguno de los anteriores cambios afecta los resultados cuando se toman en cuenta los factores de expansión. Por último, en el 2003 existen observaciones para la zona rural de San Andrés que fueron eliminados para hacerla comparable con la de 1997, pues esta última no las tenía. 


\section{Resultados}

Los resultados muestran un aumento de cobertura de aseguramiento que no es suficiente ni homogéneo entre distintos grupos de población. Se evidencia un aumento en la prevención y un mayor acceso a los medicamentos, pero una disminución en la consulta de las personas que se sintieron enfermas en el último mes principalmente causada por la falta de dinero. El gasto en medicamentos también disminuyó.

\section{Cobertura}

En el período estudiado se dio una disminución de la proporción de gente no asegurada, sin embargo, las diferencias regionales todavía subsisten. Algunos utilizan planes complementarios de salud para sustituir el servicio médico del sistema, no obstante, esta proporción es pequeña y tiene una tendencia decreciente. Los trabajadores informales y las personas que viven en las áreas rurales tienen una alta probabilidad de estar no asegurados. Cuanto menor sea el quintil de ingresos mayor es la proporción de gente afiliada al régimen subsidiado o no asegurada; mientras que la tendencia correspondiente al régimen contributivo se comporta de forma contraria.

\section{- Tipo de afiliación}

Se observa que para 2003, el $62 \%$ de la población colombiana está asegurada, el 39\% de la población total se encuentra en el régimen contribu- tivo y $23 \%$ en el régimen subsidiado, mientras que el $38 \%$ sigue sin aseguramiento en salud. El régimen subsidiado tuvo el mayor aumento de afiliados pues en 1997 representaba el 19\% de la población mientras el régimen contributivo creció en un $1 \%$.

El sistema de salud colombiano se propone, desde sus inicios, la universalidad del seguro de salud. Con la finalidad de garantizar la cobertura de los grupos de menores recursos se crea el régimen subsidiado, en el cual se prevé una concentración de los primeros quintiles, mientras que en el régimen contributivo predomina la afiliación de las personas con capacidad de pago. Como era de esperarse, la proporción de población asegurada en el régimen subsidiado disminuye al aumentar el ingreso y en el régimen contributivo aumenta. Es de resaltar que más de la mitad de la población en el quintil 1 no está asegurada y que en los quintiles más altos aparece población afiliada al régimen subsidiado. Además, en la Tabla 1, se puede observar que el porcentaje de afiliados en el régimen contributivo disminuyó en 2003 en los quintiles 1, 2, 3 y 4; por el contrario, el porcentaje de afiliados al régimen subsidiado aumentó de manera significativa en todos los quintiles.

El análisis revela las grandes diferencias en cobertura entre las regiones, donde el Pacífico muestra el mayor atraso en cobertura (Tabla 2). Por su parte, mientras en regiones como la Atlántica y Bogotá hubo un incremento alrededor de $13 \%$; en regiones como Valle y Amazonas el aumento en la cobertura fue de $4 \%$. Las regiones

Tabla 1

Tipo de afiliación según quintiles. Porcentaje y número de personas. 1997 y 2003.

\begin{tabular}{|c|c|c|c|c|c|c|c|}
\hline \multicolumn{2}{|c|}{ Quintiles } & \multicolumn{3}{|c|}{1997} & \multicolumn{3}{|c|}{2003} \\
\hline & & Contributivo & Subsidiado & No asegurado & Contributivo & Subsidiado & No asegurado \\
\hline \multirow[t]{2}{*}{1} & $\%$ & 6,4 & 35,5 & 58,0 & 6,1 & 40,5 & 53,5 \\
\hline & $\mathrm{n}$ & 608.203 & 3.358 .239 & 5.483 .603 & 529.823 & 3.540 .375 & 4.675 .604 \\
\hline \multirow[t]{2}{*}{2} & $\%$ & 20,7 & 27,0 & 52,3 & 16,2 & 36,3 & 47,5 \\
\hline & $\mathrm{n}$ & 1.834 .407 & 2.390 .340 & 4.632 .695 & 1.417 .648 & 3.172 .051 & 4.153 .918 \\
\hline \multirow[t]{2}{*}{3} & $\%$ & 41,8 & 16,4 & 41,7 & 36,1 & 22,1 & 41,8 \\
\hline & $\mathrm{n}$ & 3.290 .528 & 1.292 .882 & 3.281 .612 & 3.159 .479 & 1.933 .910 & 3.662 .085 \\
\hline \multirow[t]{2}{*}{4} & $\%$ & 62,5 & 6,2 & 31,4 & 57,4 & 11,9 & 30,7 \\
\hline & $\mathrm{n}$ & 4.404 .388 & 435.717 & 2.212 .894 & 5.012 .089 & 1.040 .200 & 2.681 .289 \\
\hline \multirow[t]{2}{*}{5} & $\%$ & 75,0 & 3,1 & 21,9 & 78,9 & 3,8 & 17,3 \\
\hline & $\mathrm{n}$ & 4.935 .457 & 204.372 & 1.438 .568 & 6.891 .345 & 335.259 & 1.512 .862 \\
\hline \multirow[t]{2}{*}{ Total } & $\%$ & 37,9 & 19,3 & 42,8 & 38,9 & 22,9 & 38,2 \\
\hline & $\mathrm{n}$ & 15.072 .983 & 7.681 .550 & 17.049 .372 & 17.008 .324 & 10.020 .069 & 16.687 .000 \\
\hline
\end{tabular}

Fuente: Encuestas de Calidad de Vida, 1997-2003 (cálculos propios). 
Tabla 2

Tipo de afiliación según región. Porcentaje y número de personas. 1997 y 2003.

\begin{tabular}{|c|c|c|c|c|c|c|c|}
\hline \multirow[t]{2}{*}{ Región } & & \multicolumn{3}{|c|}{1997} & \multicolumn{3}{|c|}{2003} \\
\hline & & Contributivo & Subsidiado & No asegurado & Contributivo & Subsidiado & No asegurado \\
\hline \multirow[t]{2}{*}{ Atlántico } & $\%$ & 28,5 & 14,4 & 57,2 & 29,5 & 26,0 & 44,5 \\
\hline & $\mathrm{n}$ & 2.426 .444 & 1.224 .153 & 4.871 .437 & 2.799 .014 & 2.472 .596 & 4.225 .841 \\
\hline \multirow[t]{2}{*}{ Oriental } & $\%$ & 32,9 & 32,4 & 34,8 & 37,1 & 28,6 & 34,3 \\
\hline & $\mathrm{n}$ & 2.265 .228 & 2.230 .951 & 2.394 .072 & 2.931 .422 & 2.259 .592 & 2.704 .706 \\
\hline \multirow[t]{2}{*}{ Central } & $\%$ & 30,4 & 21,4 & 48,2 & 30,1 & 23,2 & 46,7 \\
\hline & $\mathrm{n}$ & 1.565 .677 & 1.101 .497 & 2.476 .717 & 1.643 .327 & 1.266 .488 & 2.546 .442 \\
\hline \multirow[t]{2}{*}{ Pacífico } & $\%$ & 24,4 & 25,4 & 50,3 & 17,1 & 35,5 & 47,5 \\
\hline & $\mathrm{n}$ & 858.473 & 895.793 & 1.771 .625 & 588.922 & 1.224 .250 & 1.639 .538 \\
\hline \multirow[t]{2}{*}{ Bogotá } & $\%$ & 58,5 & 7,0 & 34,6 & 63,0 & 14,5 & 22,4 \\
\hline & $\mathrm{n}$ & 3.696 .555 & 440.257 & 2.187 .444 & 4.325 .684 & 997.803 & 1.538 .013 \\
\hline \multirow[t]{2}{*}{ Antioquia } & $\%$ & 44,9 & 19,9 & 35,1 & 45,0 & 19,1 & 35,9 \\
\hline & $\mathrm{n}$ & 2.366 .732 & 1.050 .380 & 1.849 .953 & 2.522 .940 & 1.072 .043 & 2.013 .846 \\
\hline \multirow[t]{2}{*}{ Valle } & $\%$ & 45,9 & 16,9 & 37,2 & 45,0 & 13,1 & 41,8 \\
\hline & $\mathrm{n}$ & 1.670 .756 & 613.581 & 1.354 .255 & 1.977 .125 & 576.252 & 1.836 .109 \\
\hline \multirow[t]{2}{*}{ San Andrés } & $\%$ & 68,4 & 11,4 & 20,2 & 64,6 & 19,1 & 16,3 \\
\hline & $\mathrm{n}$ & 32.454 & 5.390 & 9.586 & 37.098 & 10.975 & 9.346 \\
\hline \multirow[t]{2}{*}{ Amazonas } & $\%$ & 42,9 & 26,9 & 30,2 & 37,4 & 28,1 & 34,5 \\
\hline & $n$ & 190.664 & 119.548 & 134.283 & 186.189 & 139.997 & 172.024 \\
\hline \multirow[t]{2}{*}{ Total } & $\%$ & 37,9 & 19,3 & 42,8 & 38,9 & 22,9 & 38,2 \\
\hline & $\mathrm{n}$ & 15.072 .983 & 7.681 .550 & 17.049 .372 & 17.011 .720 & 10.019 .994 & 16.685 .864 \\
\hline
\end{tabular}

Fuente: Encuestas de Calidad de Vida, 1997-2003 (cálculos propios).

con mayor porcentaje de afiliados al régimen contributivo son Bogotá y San Andrés, mientras la región Pacífica tiene la menor proporción. Las regiones Atlántica, Oriental y Bogotá exhibieron en 2003 un aumento en el porcentaje de personas afiliadas al régimen contributivo; mientras que el Pacífico, San Andrés y Amazonas muestran una disminución.

En cuanto al régimen subsidiado, la región Pacífico tuvo la mayor proporción de afiliados (47,5\% en 2003) y el mayor crecimiento. Atlántico pasó de $14,4 \%$ en 1997 a $26 \%$ en 2003 , correspondiente a 1.248.443 nuevos afiliados. Bogotá y San Andrés también mostraron un crecimiento notable en esta proporción; por el contrario, la región Oriental y el Valle evidenciaron un decrecimiento en la proporción de afiliados al régimen subsidiado.

En cuanto al tipo de afiliación según área de ubicación se observa que el porcentaje de personas afiliadas al régimen contributivo en las áreas rurales es muy poco (13,3\%) y el porcentaje de no asegurados es bastante alto (48,3\%). En contraste, en las cabeceras municipales casi la mitad de las personas se encuentran afiliadas al régimen contributivo y la proporción de no asegurados es de $34,5 \%$. En ambas zonas se da una disminución de los no asegurados del orden del $4 \%$, esta disminución es originada principalmente por un aumento en la población afiliada al régimen subsidiado.

Es necesario hacer énfasis en que las redes de protección social en Colombia suelen estar diseñadas para un sector formal, cuyos ingresos le permiten acceder a los servicios sociales necesarios para lograr estándares de vida óptimos ${ }^{3}$. Es necesario evidenciar las fisuras que representan, en este esquema, los grupos menos favorecidos de la población. De esta forma, observar a qué sistemas de protección están acudiendo los individuos que no poseen la suficiencia monetaria ni pertenecen a un sector laboral formal es imperativo en nuestro análisis.

Para 2003, el 20,5\% de los ocupados pertenecían al régimen subsidiado (15,4\% en 1997), $44,4 \%$ al contributivo ( $45,4 \%$ en 1997$)$ y $35,1 \%$ eran no asegurados (valor cercano al 39\% para 1997). Además, cerca del 41,9\% de los ocupados que estaban en el régimen subsidiado en 1997 eran informales, en 2003 esta proporción equivale a $52,1 \%$. Las diferencias entre sectores laborales son abismales. El porcentaje de informales 
no asegurados se redujo de $80 \%$ a $68,6 \%$ por el aumento del porcentaje de informales en el régimen subsidiado. Por su parte, en 1997, cerca del $21 \%$ de los trabajadores formales no estaban asegurados, este porcentaje disminuyó a 17,9\% en el 2003.

\section{- Planes complementarios de salud}

Para complementar el análisis es conveniente evaluar a qué otros mecanismos de atención de salud se vinculan los trabajadores. Entre estos planes se encuentran las pólizas de hospitalización y cirugía, medicina prepagada, seguros estudiantiles, entre otros. Estos planes muchas veces incentivan que la gente no se afilie formalmente al sistema.

Las ECV03 muestran que cerca del 10\% de las personas afiliadas al régimen contributivo tienen un plan adicional de salud. Esto se da principalmente porque no están satisfechos con la atención y calidad de las entidades prestadoras de servicio ${ }^{4}$. Por su parte, poco más del $4 \%$ de los no asegurados tiene un plan adicional de salud y en su mayoría pertenecen a quintiles altos que prefieren asistir a médicos particulares. En ambos casos esta proporción aumenta ligeramente entre 1997 y 2003. Existe también un porcentaje de personas no mayor al $2 \%$ que dice pertenecer al régimen subsidiado y que además cuentan con planes de salud complementarios; estos dos últimos grupos podrían tener la capacidad de pago para afiliarse al régimen contributivo, pero no lo hacen.

Por regiones se observa que Bogotá, la región Oriental y el Valle son las regiones con el más alto porcentaje de afiliados a un plan de salud (alrededor de 9\%); mientras regiones como la Central, Amazonas, Pacífico y San Andrés muestran un porcentaje cercano al $2 \%$. Únicamente las regiones Oriental, Pacífica y Valle muestran un aumento en esta proporción.

Al comparar el volumen de individuos que poseen planes de salud se observa que tanto para 1997 como para 2003 los trabajadores formales se afilian más a estos servicios que los informales (en el 2003, 1,3\% contra 5\%). Esa afiliación ha disminuido entre 1997 y 2003, tanto para los trabajadores formales como para los informales, el porcentaje en 1997 para los informales era de $1,8 \%$ y para los formales de $6,4 \%$.

\section{Demanda de servicios de salud}

La demanda de servicios de salud puede examinarse a través de la demanda por prevención, la demanda por consulta ante un problema de salud y la demanda por medicamentos. Los re- sultados de prevención son positivos, pues hubo un aumento considerable. La demanda por consultas médicas ha disminuido y la falta de dinero aparece como la razón más importante. La afiliación juega un rol definitivo en el acceso a las medicinas.

\section{- Prevención}

El sistema de salud colombiano fue diseñado con especial atención a la promoción y prevención de la salud a las cuales destinó explícitamente un porcentaje de la Unidad de Pago por Capitación (UPC). Efectivamente, las encuestas muestran un aumento del porcentaje de población asegurada que consulta por prevención; sin embargo, este aumento es mayor en la población asegurada en el régimen contributivo (de $59 \%$ a $71 \%$ ), menor en el subsidiado (de $34 \%$ a $52 \%$ ) y no se da en los no asegurados. Parecería razonable atribuir este hecho a la expedición de la resolución 412 que estableció, aun cuando con muchas limitaciones, la demanda inducida en una serie de actividades preventivas.

\section{- Consulta}

Luego de indagar si el individuo presentó problemas de salud en el último mes, la encuesta analiza la acción que tomaron ante el problema. Entre estas opciones se encuentra acudir a un médico, a un promotor de salud, a un boticario, consultar a un curandero, terapias alternativas, remedios caseros, autoprescripción o nada. Las encuestas muestran una disminución del porcentaje de personas que van al médico, para 1997 esta proporción era de 77,1\% y para 2003 fue de $67,9 \%$. Por el contrario, las personas reportan acudir más al boticario, utilización de remedios caseros y la autoprescripción.

El único régimen que ha incrementado la atención de las personas con problemas de salud es el subsidiado, que aumentó en 4 puntos la proporción de pacientes atendidos por profesionales médicos, siendo este porcentaje de $74,4 \%$ en 2003. En el régimen contributivo esta proporción decreció en 8 puntos porcentuales llegando $79,5 \%$ en 2003, claramente una caída muy significativa. Los no asegurados son quienes presentan la más fuerte disminución de atención médica profesional, alrededor de 20 puntos, llegando en 2003 a 49,7\%. Como se argumenta después, esto se debe, en su mayoría, a la falta de dinero; lo que muestra uno de los grandes beneficios de estar asegurado al disminuir el riesgo de caer enfermo.

Dado que una proporción importante no asiste al médico, es necesario conocer las razones 
por las que toman esta decisión. Al respecto, la falta de dinero se revela como la principal barrera de acceso al servicio médico (39,3\% en 2003). Para los quintiles más bajos la falta de dinero es, en más de la mitad de los casos, la causa para no ir al médico. Esta proporción disminuye sensiblemente cuanto mayor sea el quintil, y en 2003 representa el 13,3\% en el quintil más alto.

Cuando se comparan los regímenes con las razones de no atención se encuentra que en el régimen contributivo la falta de dinero aumentó preocupantemente, pues pasó de 5,5\% a 10,6\%. Por el contrario, las personas pertenecientes al régimen subsidiado reportaron una importante disminución de la falta de dinero como barrera de acceso, pasando de $41,4 \%$ a $29,5 \%$. Por su parte, más de la mitad de las personas que no están aseguradas y no recibieron atención médica señalan la falta de dinero como impedimento para acceder a los servicios médicos, sin presentar cambios importantes entre los dos años del análisis.

\section{- Medicamentos}

El gasto en medicamentos se ha destacado como uno de los mayores rubros del gasto en salud de los hogares en los últimos años, Ramírez et al. 5 muestran que en 1997 el 24,95\% del gasto en salud y el $2,4 \%$ del ingreso se gasta en medicamentos. Con el fin de conocer el uso de medicamentos por parte de la población se tomó la población que reportó haber estado enferma en los últimos treinta días y que usó medicamentos para tratarla.

Las encuestas muestran que el $75,1 \%$ de la población usó medicamentos en 2003 cuando estuvo enferma. Este porcentaje disminuyó para los no asegurados entre 1997 y 2003 al pasar de $75,9 \%$ a $66,7 \%$, mientras aumentó considerablemente para las personas del régimen subsidiado pasando de $72,4 \%$ a $78,3 \%$. Para las personas del régimen contributivo no se dan cambios importantes presentando en 2003 un porcentaje de $79,3 \%$. De esta forma también se muestra un mayor acceso a los medicamentos por parte de los asegurados en 2003 que se ve apoyado por una mayor entrega de medicamentos por las prestadoras de servicio, aunque éste aún dista de ser perfecto. En 2003, el 2,9\% de los no asegurados recibe por lo menos algún medicamento; por el contrario, en el régimen contributivo este porcentaje es cercano al $74,4 \%$ y en el subsidiado es del 67,6\%. En 1997 estos porcentajes eran de 0,8\%, $68,6 \%$ y $50,1 \%$, respectivamente, observándose un mayor aumento en el régimen subsidiado. De esta manera, se encuentra que la afiliación al sistema juega un papel importante en el uso de medicinas y en la entrega de ellas por parte de las instituciones prestadoras de servicio.

Lo anterior tiene implicaciones directas sobre el gasto que realizan los hogares en medicamentos. El gasto per cápita en medicamentos en el régimen contributivo es similar en los dos años del análisis, alrededor de $\$ 14.000$. Por otro lado, el gasto de los no afiliados y de las personas afiliadas al régimen subsidiado disminuye en 2003. Para los primeros pasa de $\$ 8.500$ a $\$ 6.500$ y para los segundos pasó de $\$ 13.000$ a $\$ 9.500$, aproximadamente.

\section{Conclusiones y consideraciones}

Este trabajo muestra los principales avances en cuanto a aseguramiento y demanda de servicios de salud entre los años 1997 y 2003 a partir de las encuestas de calidad de vida. Se observó un aumento en el aseguramiento en salud de la población, aunque esta sigue siendo insuficiente y heterogénea entre los distintos sectores de la población. El análisis muestra que existen falencias en la cobertura de la población más vulnerable y no es sostenible para las personas que poseen un trabajo informal.

Este aumento de aseguramiento se debió al incremento de la afiliación al régimen subsidiado. El régimen contributivo presenta un estancamiento que puede ser causado por la inestabilidad económica del país, pero que también puede ser debido a los incentivos del sistema para afiliarse a este régimen. Desde la reforma de la Ley 100 el desempleo, la informalidad y la pobreza presentaron tendencias crecientes. Aunque estos fenómenos son multicausales, una parte de la explicación puede deberse a que la afiliación de los empleados de una empresa al sistema elevó los costos de producción 6 , esto lleva a menores salarios (pobreza), a la disminución de la plantilla de trabajadores (desempleo) o a contratarlos como trabajadores independientes (informalidad).

El hecho de que el aumento de cobertura no pueda darse a través del mercado y sea únicamente a través de la intervención del estado implica un gran esfuerzo financiero que no es sostenible porque parte de esos recursos provienen del régimen contributivo, que está estancado, y la otra parte del Sistema General de Participaciones que depende del desempeño de la economía, de la capacidad de tributación o del endeudamiento, que tampoco es favorable en ningún caso. Esto ya ha sido reconocido y ha llevado a una afiliación con un plan de beneficios todavía más pequeño (subsidios parciales), lo que origina un mayor fraccionamiento de los planes de atención y un acceso más restringido a los servicios de salud. 
Aun cuando se ha dicho que el aseguramiento no es salud, sí ofrece ventajas sobre el acceso a los servicios de salud. Como lo sugieren Restrepo et al. 7 , la consulta al médico es altamente dependiente del régimen de afiliación y los no asegurados son los que tienen una menor probabilidad de ir al médico cuando se sienten enfermos. Al investigar las causas de la inasistencia al médico se encuentra que la principal razón es la falta de dinero; dada la reciente crisis económica que golpeó fuertemente a los hogares ${ }^{8}$, el porcentaje de personas que no asistió al médico es mayor para el 2003.

La consulta por prevención para 2003 fue mayor que en 1997. El porcentaje de personas que consultan por prevención también es mayor si están asegurados. También se encuentra que el régimen al que pertenece el individuo es determinante en el momento de acceder a los medicamentos cuando se está enfermo, siendo el régimen contributivo aquel que presenta el mejor indicador.

\section{Resumen}

En este documento se esbozan los principales avances en cuanto a aseguramiento en salud y demanda de servicios médicos en Colombia, a partir de las Encuestas de Calidad de Vida de 1997 y 2003. Se encuentra un aumento en el aseguramiento en salud de la población, con un mayor incremento del régimen subsidiado; aún así, persiste una gran proporción que no cuenta con aseguramiento. También se encuentra que el tipo de afiliación afecta directamente la demanda de servicios. Se evidencia un aumento en la consulta por prevención para 2003 y una disminución en las consultas médicas cuando el individuo se siente enfermo, esta disminución se debe principalmente a la falta de dinero. El acceso a los medicamentos aumenta entre 1997 y 2003, lo que se refleja en la disminución del gasto privado en medicamentos.

Cobertura de Servicios de Salud; Necesidades y Demandas de Servicios de Salud; Calidad de Vida
Lo anterior muestra que el aseguramiento es importante para el acceso a servicios médicos, el problema es que el aseguramiento está estancado. El sistema debe reformarse de tal forma que se ofrezcan mejores incentivos para reactivar la dinámica del mercado laboral lo que puede llevar a aumentos del aseguramiento en el régimen contributivo. Esto, a su vez, generará más recursos para ampliar los recursos de afiliación al régimen subsidiado. También debe asegurarse un crecimiento sostenible y equitativo del ingreso de las personas pues, como se mostró, es la principal barrera de acceso de los hogares.

Además, mejores políticas de prevención, incluso para aquellos que no están afiliados, mayor educación de las personas y una mejor calidad de los servicios pueden aumentar la demanda por los servicios médicos. También se debe trabajar para forzar a que las instituciones prestadoras de servicios entreguen oportunamente los medicamentos y no impongan barreras al acceso de éstos.

\section{Colaboradores}

A. Zambrano fue el encargado de coordinar el equipo de trabajo, del análisis e interpretación de los datos y de la escritura de las conclusiones y consideraciones. M. Ramírez y F. J. Yepes son los codirectores del proyecto de investigación del cual subyace este documento. Ambos diseñaron el estudio, realizaron varias revisiones críticas del documento y enunciaron distintos aportes para la consolidación de las conclusiones. J. A. Guerra y D. Rivera estuvieron a cargo del análisis e interpretación de datos y en la escritura de las distintas secciones.

\section{Agradecimientos}

Este documento es producto del proyecto de investigación Governance and Evidence Based Decision Making: A Participatory Formation Process of Health Policies financiado por el Internacional Development Research Centre (IDRC), realizado conjuntamente entre la Asociación Colombiana de la Salud (ASSALUD) y la Facultad de Economía de la Universidad del Rosario; y del proyecto Análisis de los Resultados de la Encuesta de Calidad de Vida 2003: Alcance de los Programas Estatales y Servicios Públicos en Hogares y Personas financiado por el DANE, realizado por la Facultad de Economía de la Universidad del Rosario. Agradecemos la colaboración y comentarios de Olga Lucía Acosta, Jairo Humberto Restrepo, Claudio Karl y el de un evaluador anónimo. 


\section{Referencias}

1. Umaña H, Torres J. Encuesta nacional sobre conocimientos, actitudes y prácticas en salud. Seguridad social. v. I: características de la población. Bogotá: División de Investigaciones Especiales, Instituto Nacional de Salud, Ministerio de Salud; 1990.

2. Departamento Administrativo Nacional de Estadística. Encuesta de calidad de vida 2003 - manual de recolección y conceptos básicos. Bogotá: Departamento Administrativo Nacional de Estadística; 2003.

3. Acosta OL, Ramírez M, Cañón C. Principales estudios sobre sostenibilidad financiera del sistema general de seguridad social en salud. Bogotá: Fundación Corona/Universidad del Rosario; 2005.

4. Acosta OL, Guerra J, Rivera DM. Acceso de los hogares a los principales servicios públicos y sociales y percepciones de calidad sobre estos servicios. Bogotá: Centro Editorial Universidad del Rosario; 2005. (Borrador de Investigación, 76).

5. Ramírez M, Cortés D, Gallego J. El gasto en salud de los hogares colombianos; un análisis descriptivo. Lecturas de Economía 2002; 57:87-125.
6. Kugler A, Kugler M. Effects of payroll taxes on employment and wages: evidence from the Colombian Social Security Reform. Stanford: Stanford University; 2002. (Center for Research on Economic Development and Policy Reform Working Papers, 134).

7. Restrepo J, Zambrano A, Vélez M, Ramírez M. Health insurance as a strategy for access: streamlined facts of the Colombian Health Care Reform. In: $5^{\text {th }}$ World Congress of the International Health Economics Association. Barcelona: International Health Economics Association; 2005.

8. Ramírez M, Muñoz M, Zambrano A. Comparación del gasto de los hogares entre 1997 y 2003 según resultados de las encuestas de calidad de vida: magnitud, composición y distribución. Bogotá: Centro Editorial Universidad del Rosario; 2005. (Borrador de Investigación, 67).

Recibido el 27/Dic/2006

Versión final presentada el 19/Jul/2007

Aprobado el 24/Jul/2007 